\title{
THE HOMOLOGICAL DIMENSION OF A QUOTIENT FIELD
}

\author{
IRVING KAPLANSKY \\ In memory of Professor TADAsi Nakayama
}

Let $R$ be an integral domain, $Q$ its quotient field. For any $R$-module $A$ we write $d(A)$ for its homological dimension (minimal length of a projective resolution), or $d_{R}(A)$ if it is necessary to call attention to $R$.

The number $d(Q)$ is pertinent to several questions concerning $R$-modules [3], [4]. We summarize what is known about it. It is an easy exercise that $d(Q) \leqq 1$ if $Q$ is a countably generated $R$-module. Also [3, Lemma 3.2], $d(Q)$ $=1$ if $R$ is Noetherian of Krull dimension one and this result is generalized in [4]. In $1957 \mathrm{I}$ noted a result in the converse direction (mentioned on page 386 of [3]) : if $R$ is a valuation ring and $d(Q)=1$, then $Q$ is countably generated.

In the present note I prove a more general converse: if $d(Q)=1$ and $R$ is quasi-local (i.e. has exactly one maximal ideal) then $Q$ is a countably generated $R$-module. The basic device is similar to the artifice used in [1] and is simpler than my 1957 treatment of the valuation ring case.

We begin the discussion by letting $R$ be any integral domain, $Q$ its quotient field. Let $S$ denote the set of all elements $a^{-1}, a$ ranging over the elements of $R$ that are not zero and not units. Of course $S$ spans $Q$ as an $R$-module, and we choose to resolve $Q$ extravagantly by mapping a free module $F$ onto $Q, F$ having a basis $\left\{\boldsymbol{u}_{a}\right\}$ indexed by the elements of $R$ not zero or units, the mapping $f: F \rightarrow Q$ being given by $u_{a} \rightarrow a^{-1}$. Let the kernel of $f$ be $G$. We assume that $G$ is free (this will be the case if $R$ is quasi-local and $d(Q) \leqq 1$, since all projectives over $R$ are then free [1]). Let $\left\{v_{\alpha}\right\}$ be a basis of $G$.

Let $S_{0}$ be a countable semigroup in $S$ (i.e. a countable subset closed under multiplication). We describe a procedure that will be iterated ad infinitum. Let $F_{0}$ be the submodule of $F$ spanned by the basis elements corresponding to $S_{0}$. Let $G_{0}=G \cap F_{0}$ (i.e. the kernel of $f$ when restricted to $F_{0}$ ). It is easily

Received May 4, 1965. 
seen that $G_{0}$ is countably generated. (One way to do this: number the elements of $S_{0}$, let $b_{n}$ be the product of the first $n$, and let $u_{n}$ be the basis element with $f\left(\boldsymbol{u}_{n}\right)=b_{n}$; then the elements $u_{n}-\left(b_{n} / b_{n+1}\right) u_{n+1}$ span $\left.G_{0}\right)$. Pick a countable generation of $G_{0}$, write each generator as a linear combination of $v^{\prime} s$, write each resulting $v$ as a linear combination of $u^{\prime} s$, and assemble the elements $f(u)$ for these $u^{\prime} s$. Together with $S_{0}$, these $f(u)^{\prime} s$ will generate a new countable semi-group $S_{1}$.

Iteration of the above procedure yields an increasing sequence of countable semigroups $S_{0}, S_{1}, S_{2}, \ldots$ Let $S^{*}$ denote their union. Then $S^{*}$ is again a countable sub-semi-group of $S$. When we (as done above for $S_{0}$ ) write $F^{*}$ for the submodule of $F$ spanned by the basis elements corresponding to $S^{*}$, and $G^{*}=F^{*} \cap G$, we find that $G^{*}$ is a free direct summand of $G$, being spanned by the totality of $v^{\prime} s$ that arose in the construction.

Let $A$ be the $R$-submedule of $Q$ spanned by $S^{*}$. Let $B$ be any $R$-module lying between $A$ and $Q$ that is spanned by reciprocals of elements of $R$. Denote by $C$ the submodule of $F$ spanned by the elements $u_{a}$ corresponding to all elements $a^{-1}$ lying in $B$. Let $D=G \cap C$. The exact sequence

$$
0 \rightarrow D \rightarrow C \rightarrow B \rightarrow 0
$$

furnishes a short free resolution of $B$. In the application shortly to be made, the module $B$ will be non-projective; hence we will have

$$
d(D)=d(B)-1 \text {. }
$$

Next we note the induced free resolution of $B / A$ :

$$
0 \rightarrow D / G^{*} \rightarrow C / F^{*} \rightarrow B / A \rightarrow 0 .
$$

In the forthcoming application it will also be the case that $B / A$ is non-projeotive. But even if $B / A$ is projective we always have the inequality

$$
d\left(D / G^{*}\right) \geqq d(B / A)-1 .
$$

Since $G^{*}$ is a direct summand of $G$, it is also a direct summand of the intermediate $D$. Thus $D$ is the direct sum of $D / G^{*}$ and the free module $G^{*}$. Hence

$$
d(D)=d\left(D / G^{*}\right) .
$$

On putting together (1), (2), and (3) we find 


$$
d(B / A) \leqq d(B)
$$

The analysis up to this point may be useful in future generalizations, but we turn specifically now to the proof of our main result.

Theorem 1. Let $R$ be a quasi-local integral domain with quotient field $Q$, and assume $d(Q) \leqq 1$. Then $Q$ is a countably generated $R$-module.

Proof. We construct $S^{*}$ and $A$ as above. (An intial $S_{0}$ is needed; it can be taken to consist of the powers of any element of $S$ ). We shall prove $A=Q$ by deriving a contradiction from the contrary assumption. If $A \neq Q$ we pick $q \in R$ (not 0 or a unit) such that $q^{-1}$ is not in $A$. Our choice for $B$ is $q^{-1} A$. We have that $B$ properly contains $A$, and also that $B$ is isomorphic to $A$. The module $A$ is spanned by a sequence of elements each of which is a proper multiple of the succeeding one. From this we readily deduce $d(A)=1$, and so we likewise have $d(B)=1$. In particular, $B$ is not projective; the discussion above is thus applicable and we have that (4) holds. Hence $d(B / A) \leqq 1$. But we now argue that $d(B / A)=2$.

The argument is based on two lemmas on homological dimension which are perhaps most easily quoted from [2]. Let $T$ denote the ring $R /(q)$. We note that $B / A=B / q B$. Then [2, Th. 1.7] $d_{T}(B / A) \leqq d_{R}(B)=1$. The possibility that $d_{T}(B / A)=0$ is readily excluded. For suppose that $B / A$ is free as a $T$ module. We have a sequence $u_{i}$ of generators of $B / A$ with the property $u_{i}=$ $\lambda_{i} u_{i+1}$, where $\lambda_{i}$ is a non-unit in $T$. For large enough $i$ it must be the case that $u_{i}$ has (in its expression in terms of a $T$-basis of $B / A$ ) a unit for one of its coefficients. But then the equation $u_{i}=\lambda_{i} u_{i+1}$ is impossible at that coordinate.

Hence $d_{T}(B / A)=1$. The hypotheses of [2, Th. 1.3] are fulfilled and we deduce $d(B / A)=2$. With this contradiction the proof of Theorem 1 is complete.

Theorem 1 cannot be extended automatically to the global case, as is shown by the case of Noetherian domains of Krull dimension one. Perhaps in some sense this is the only exception. We can at any rate handle the case of polynomial rings over a field.

Theorem 2. Let $K$ be an uncountable field and $R$ a polynomial ring over $K$ in $n$ indeterminates, $n \geqq 2$. Let $Q$ be the quotient field of $R$. Then $d(Q) \geqq 2$. If $n=2, d(Q)=2$. 
Proof. Let $M$ denote the maximal ideal of $R$ at the origin. We have that $Q$ is also the quotient field of the localization $R_{M}$, and that $Q=Q_{\mathcal{M}}$. Thus $d R_{\mu l}(Q) \leqq d_{R}(Q)$. Now $R_{\mu}$ is a unique factorization domain which has uncountably many primes (if $x$ and $y$ are two of the indeterminates and $\alpha$ ranges over $K$, the elements $x+\alpha y$ are distinct primes in $R_{M}$ ). This means that the quotient field of $R_{M}$ is not a countably generated $R_{M}$-module. By Theorem $1, d R_{M}(Q) \geqq$ 2 , and so $d_{R}(Q) \geqq 2$. The final statement of the theorem is evident since for $n=2$ the global dimension of $R$ is 2 .

I am indebted to $\mathrm{H}$. Bass for a spirited discussion that resulted in several significant improvements.

\section{BIBLIOGRAPHY}

[1] I. Kaplansky, Projective modules, Annals of Math. 68 (1958), 372-7.

[2] I. Kaplansky, Homological dimension of rings and modules, Univ. of Chicago mimeographed notes, 1959.

[3] E. Matlis, Divisible modules, Proc. Amer Math. Soc. 11 (1960), 385-391.

[4] E. Matlis, Cotorsion modules, Memoirs Amer. Math. Soc. no. 49, 1964.

University of Chicago 